\title{
Extended central factorial polynomials of the second kind
}

\author{
Taekyun Kim¹, Dae San Kim², Gwan-Woo Jang ${ }^{1}$ and Jongkyum Kwon ${ }^{3 *}$
}

\section{${ }^{*}$ Correspondence:}

mathkjk26@gnu.ac.kr

${ }^{3}$ Department of Mathematics

Education and ERI, Gyeongsang

National University, Jinju, Republic of Korea

Full list of author information is available at the end of the article

\begin{abstract}
In this paper, we consider the extended central factorial polynomials and numbers of the second kind, and investigate some properties and identities for these polynomials and numbers. In addition, we give some relations between those polynomials and the extended central Bell polynomials. Finally, we present some applications of our results to moments of Poisson distributions.
\end{abstract}

MSC: $11 \mathrm{~B} 75 ; 11 \mathrm{~B} 83$

Keywords: Extended central factorial polynomials of the second kind; Extended central Bell polynomials

\section{Introduction}

For $n \geq 0$, the central factorial numbers of the second kind are defined by

$$
x^{n}=\sum_{k=0}^{n} T(n, k) x^{[k]}, \quad n \geq 0,(\text { see }[1,2,20-22])
$$

where $x^{[k]}=x\left(x+\frac{k}{2}-1\right)\left(x+\frac{k}{2}-2\right) \cdots\left(x-\frac{k}{2}+1\right), k \geq 1, x^{[0]}=1$.

By (1.1), we see that the generating function of the central factorial numbers of the second kind is given by

$$
\frac{1}{k !}\left(e^{\frac{t}{2}}-e^{-\frac{t}{2}}\right)^{k}=\sum_{n=k}^{\infty} T(n, k) \frac{t^{n}}{n !} \quad(\text { see }[1-4,21]) .
$$

Here the definition of $T(n, k)$ is extended so that $T(n, k)=0$ for $n<k$. This agreement will be applied to all similar situations without further mention.

Then, by (1.2), we have

$$
T(n, k)=\frac{1}{k !} \sum_{j=0}^{k}\left(\begin{array}{l}
k \\
j
\end{array}\right)(-1)^{k-j}\left(j-\frac{k}{2}\right)^{n}, \quad n, k \geq 0 .
$$

\section{照 Springer}


Let us recall that the Stirling polynomials of the second kind are defined by

$$
\frac{1}{k !} e^{x t}\left(e^{t}-1\right)^{k}=\sum_{n=k}^{\infty} S_{2}(n, k \mid x) \frac{t^{n}}{n !} \quad(\text { see }[10,16,17])
$$

where $k$ is a nonnegative integer.

When $x=0, S_{2}(n, k)=S_{2}(n, k \mid 0), n, k \geq 0$, are the Stirling numbers of the second kind given by

$$
x^{n}=\sum_{k=0}^{n} S_{2}(n, k)(x)_{k}, \quad n \geq 0(\text { see }[12,14,15,21]),
$$

where $(x)_{0}=1,(x)_{k}=x(x-1) \cdots(x-k+1), k \geq 1$.

From (1.4), we note that

$$
\begin{aligned}
S_{2}(n, k \mid x) & =\sum_{l=k}^{n}\left(\begin{array}{l}
n \\
l
\end{array}\right) S_{2}(l, k) x^{n-l} \\
& =\frac{1}{k !} \sum_{j=0}^{k}\left(\begin{array}{l}
k \\
j
\end{array}\right)(-1)^{k-j}(j+x)^{n}, \quad n, k \geq 0 .
\end{aligned}
$$

The Bell polynomials are given by the generating function

$$
e^{x\left(e^{t}-1\right)}=\sum_{n=0}^{\infty} \operatorname{Bel}_{n}(x) \frac{t^{n}}{n !} \quad(\text { see }[5-11])
$$

Then, from (1.4) and (1.6), we get

$$
\operatorname{Bel}_{n}(x)=\sum_{m=0}^{n} S_{2}(n, m) x^{m} \quad(\text { see }[10])
$$

In [17], the extended Stirling polynomials of the second kind are defined by

$$
\frac{1}{k !} e^{x t}\left(e^{t}-1+r t\right)^{k}=\sum_{n=k}^{\infty} S_{2, r}(n, k \mid x) \frac{t^{n}}{n !}
$$

where $n, k \in \mathbb{N} \cup\{0\}$ and $r \in \mathbb{R}$.

When $x=0, S_{2, r}(n, k)=S_{2, r}(n, k \mid 0), n, k \geq 0$, are called the extended Stirling numbers of the second kind. Note that $S_{2,0}(n, k)=S_{2}(n, k)$ and $S_{2,0}(n, k \mid x)=S_{2}(n, k \mid x)$.

From (1.4) and (1.8), we note that

$$
S_{2, r}(n, k)=\sum_{l=0}^{k}\left(\begin{array}{l}
n \\
l
\end{array}\right) r^{l} S_{2}(n-l, k-l), \quad n, k \geq 0 \text { (see [17]). }
$$

It is known that the extended Bell polynomials are defined by

$$
e^{x\left(e^{t}-1+r t\right)}=\sum_{n=0}^{\infty} \operatorname{Bel}_{n, r}(x) \frac{t^{n}}{n !} \quad(\text { see }[13,18,19]) .
$$

For $x=1, \operatorname{Bel}_{n, r}=\operatorname{Bel}_{n, r}(1)$ are called the extended Bell numbers. 
Then, from (1.10), we get

$$
\operatorname{Bel}_{n, r}(x)=\sum_{m=0}^{n} x^{m} S_{2, r}(n, m) \quad(\text { see [17]). }
$$

Recently, the central Bell polynomials were defined by Kim as

$$
\sum_{n=k}^{\infty} \operatorname{Bel}_{n}^{(c)}(x) \frac{t^{n}}{n !}=e^{x\left(e^{\frac{t}{2}}-e^{-\frac{t}{2}}\right)} \quad(\text { see }[1-3])
$$

For $x=1, \mathrm{Bel}_{n}^{(c)}=\mathrm{Bel}_{n}^{(c)}(1)$ are called the central Bell numbers.

Thus, by (1.12), we get

$$
\operatorname{Bel}_{n}^{(c)}(x)=\sum_{k=0}^{n} x^{k} T(n, k), \quad n \geq 0(\text { see }[17])
$$

The purpose of this paper is to consider the extended central factorial polynomials and numbers of the second kind, and investigate some properties and identities for these polynomials and numbers. In addition, we give some relations between those polynomials and the extended central Bell polynomials. Finally, we present some applications of our results to moments of Poisson distributions.

\section{Extended central factorial polynomials of the second kind}

Motivated by (1.8), we define the extended central factorial polynomials of the second kind by

$$
\frac{1}{k !} e^{x t}\left(e^{\frac{t}{2}}-e^{-\frac{t}{2}}+r t\right)^{k}=\sum_{n=k}^{\infty} T^{(r)}(n, k \mid x) \frac{t^{n}}{n !},
$$

where $k \in \mathbb{N} \cup\{0\}$ and $r \in \mathbb{R}$. When $x=0, T^{(r)}(n, k)=T^{(r)}(n, k \mid 0), n, k \geq 0$, are called the extended central factorial numbers of the second kind. Note here that, when $r=0$, $T(n, k \mid x)=T^{(0)}(n, k \mid x)$ and $T(n, k)=T^{(0)}(n, k)$ are respectively the central factorial polynomials of the second kind and the central factorial numbers of the second kind.

From (2.1), we note that

$$
\begin{aligned}
\sum_{n=k}^{\infty} T^{(r)}(n, k \mid x) \frac{t^{n}}{n !} & =\sum_{m=0}^{\infty} \frac{1}{m !} x^{m} t^{m} \sum_{l=k}^{\infty} T^{(r)}(l, k) \frac{t^{l}}{l !} \\
& =\sum_{n=k}^{\infty}\left(\sum_{l=k}^{n}\left(\begin{array}{l}
n \\
l
\end{array}\right) T^{(r)}(l, k) x^{n-l}\right) \frac{t^{n}}{n !}
\end{aligned}
$$

Therefore, by comparing the coefficients on both sides of (2.2), we obtain the following theorem.

Theorem 2.1 For $n, k \geq 0$, we have

$$
T^{(r)}(n, k \mid x)=\sum_{l=k}^{n}\left(\begin{array}{l}
n \\
l
\end{array}\right) T^{(r)}(l, k) x^{n-l} .
$$


From (2.1), we note that

$$
\begin{aligned}
\sum_{k=0}^{\infty} \frac{x^{k}}{k !}\left(e^{\frac{t}{2}}-e^{-\frac{t}{2}}+r t\right)^{k} & =\sum_{k=0}^{\infty} x^{k} \sum_{n=k}^{\infty} T^{(r)}(n, k) \frac{t^{n}}{n !} \\
& =\sum_{n=0}^{\infty}\left(\sum_{k=0}^{n} x^{k} T^{(r)}(n, k)\right) \frac{t^{n}}{n !}
\end{aligned}
$$

On the other hand,

$$
\begin{aligned}
\sum_{k=0}^{\infty} \frac{x^{k}}{k !}\left(e^{\frac{t}{2}}-e^{-\frac{t}{2}}+r t\right)^{k} & =e^{x\left(e^{\frac{t}{2}}-e^{-\frac{t}{2}}+r t\right)}=e^{x\left(e^{\frac{t}{2}}-e^{-\frac{t}{2}}\right)} e^{x r t} \\
& =\sum_{k=0}^{\infty} \operatorname{Bel}_{k}^{(c)}(x) \frac{t^{k}}{k !} \sum_{m=0}^{\infty} r^{m} x^{m} \frac{t^{m}}{m !} \\
& =\sum_{n=0}^{\infty}\left(\sum_{k=0}^{n}\left(\begin{array}{l}
n \\
k
\end{array}\right) \operatorname{Bel}_{k}^{(c)}(x) r^{n-k} x^{n-k}\right) \frac{t^{n}}{n !} .
\end{aligned}
$$

Now, we define the extended central Bell polynomials by

$$
e^{x\left(e^{\frac{t}{2}}-e^{-\frac{t}{2}}+r t\right)}=\sum_{n=0}^{\infty} \operatorname{Bel}_{n}^{(c, r)}(x) \frac{t^{n}}{n !} .
$$

For $x=1, \operatorname{Bel}_{n}^{(c, r)}=\operatorname{Bel}_{n}^{(c, r)}(1)$ are called the extended central Bell numbers.

Therefore, by combining (2.4)-(2.6), we obtain the following theorem.

Theorem 2.2 For $n \geq 0$, we have

$$
\begin{aligned}
\operatorname{Bel}_{n}^{(c, r)}(x) & =\sum_{k=0}^{n} x^{k} T^{(r)}(n, k) \\
& =\sum_{k=0}^{n}\left(\begin{array}{l}
n \\
k
\end{array}\right) \operatorname{Bel}_{k}^{(c)}(x) r^{n-k} x^{n-k} .
\end{aligned}
$$

In particular,

$$
\operatorname{Bel}_{n}^{(c, r)}=\sum_{k=0}^{n}\left(\begin{array}{l}
n \\
k
\end{array}\right) \operatorname{Bel}_{k}^{(c)} r^{n-k}=\sum_{k=0}^{n} T^{(r)}(n, k) .
$$

Remark By (2.6), we get

$$
\begin{aligned}
\sum_{n=0}^{\infty} \operatorname{Bel}_{n}^{(c, r)}(x) \frac{t^{n}}{n !} & =e^{x t} e^{x\left(e^{\frac{t}{2}}-e^{-\frac{t}{2}}+(r-1) t\right)} \\
& =\sum_{k=0}^{\infty} x^{k} \frac{1}{k !} e^{x t}\left(e^{\frac{t}{2}}-e^{-\frac{t}{2}}+(r-1) t\right)^{k}
\end{aligned}
$$




$$
\begin{aligned}
& =\sum_{k=0}^{\infty}\left(\sum_{n=k}^{\infty} T^{(r-1)}(n, k \mid x) \frac{t^{n}}{n !}\right) x^{k} \\
& =\sum_{n=0}^{\infty}\left(\sum_{k=0}^{n} x^{k} T^{(r-1)}(n, k \mid x)\right) \frac{t^{n}}{n !}
\end{aligned}
$$

Comparing the coefficients on both sides of (2.9), we get

$$
\operatorname{Bel}_{n}^{(c, r)}(x)=\sum_{k=0}^{n} x^{k} T^{(r-1)}(n, k \mid x), \quad n \geq 0
$$

In particular,

$$
\operatorname{Bel}_{n}^{(c, r)}=\sum_{k=0}^{n} T^{(r-1)}(n, k \mid 1)
$$

and, invoking (2.3),

$$
\operatorname{Bel}_{n}^{(c, 1)}(x)=\sum_{k=0}^{n} T(n, k \mid x) x^{k}=\sum_{k=0}^{n} \sum_{l=k}^{n}\left(\begin{array}{l}
n \\
l
\end{array}\right) T(l, k) x^{n-l+k}
$$

Therefore, by (2.10)-(2.12), we obtain the following corollary.

Corollary 2.3 For $n \geq 0$, we have

$$
\operatorname{Bel}_{n}^{(c, r)}(x)=\sum_{k=0}^{n} x^{k} T^{(r-1)}(n, k \mid x) .
$$

In particular,

$$
\operatorname{Bel}_{n}^{(c, r)}=\sum_{k=0}^{n} T^{(r-1)}(n, k \mid 1)
$$

and

$$
\operatorname{Bel}_{n}^{(c, 1)}(x)=\sum_{k=0}^{n} \sum_{l=k}^{n}\left(\begin{array}{l}
n \\
l
\end{array}\right) T(l, k) x^{n-l+k}
$$

From (2.1), we note that

$$
\begin{aligned}
\sum_{n=0}^{\infty} T^{(r)}(n, k) \frac{t^{n}}{n !} & =\frac{1}{k !} \sum_{l=0}^{k}\left(\begin{array}{l}
k \\
l
\end{array}\right) r^{l} t^{l}\left(e^{\frac{t}{2}}-e^{-\frac{t}{2}}\right)^{k-l} \\
& =\sum_{l=0}^{k} \frac{r^{l}}{l !} t^{l} \frac{1}{(k-l) !}\left(e^{\frac{t}{2}}-e^{-\frac{t}{2}}\right)^{k-l}
\end{aligned}
$$




$$
\begin{aligned}
& =\sum_{l=0}^{k} \frac{r^{l}}{l !} t^{l} \sum_{n=k}^{\infty} T(n-l, k-l) \frac{t^{n-l}}{(n-l) !} \\
& =\sum_{n=k}^{\infty}\left(\sum_{l=0}^{k}\left(\begin{array}{l}
n \\
l
\end{array}\right) r^{l} T(n-l, k-l)\right) \frac{t^{n}}{n !} .
\end{aligned}
$$

By comparing the coefficients on both sides of (2.13), we obtain the following theorem.

Theorem 2.4 For $n, k \geq 0$, we have

$$
T^{(r)}(n, k)=\sum_{l=0}^{k}\left(\begin{array}{l}
n \\
l
\end{array}\right) r^{l} T(n-l, k-l) .
$$

From (2.6), we note that

$$
\begin{aligned}
e^{\left(e^{\frac{t}{2}}-e^{-\frac{t}{2}}+r t\right)} & =e^{\left(e^{\frac{t}{2}}-e^{\left.-\frac{t}{2}\right)}\right.} e^{r t}=\sum_{k=0}^{\infty} \mathrm{Bel}_{k}^{(c)} \frac{t^{k}}{k !} \sum_{k=0}^{\infty} r^{l} \frac{t^{l}}{l !} \\
& =\sum_{n=0}^{\infty}\left(\sum_{k=0}^{n}\left(\begin{array}{l}
n \\
k
\end{array}\right) r^{n-k} \mathrm{Bel}_{k}^{(c)}\right) \frac{t^{n}}{n !} .
\end{aligned}
$$

Therefore, by (2.14) and (2.8), we obtain the following theorem.

Theorem 2.5 For $n \geq 0$, we have

$$
\operatorname{Bel}_{n}^{(c, r)}=\sum_{k=0}^{n} \sum_{l=0}^{k}\left(\begin{array}{l}
n \\
l
\end{array}\right) r^{l} T(n-l, k-l)
$$

From (2.1), we have

$$
\begin{aligned}
\sum_{n=k}^{\infty} T(n, k \mid x) \frac{t^{n}}{n !} & =\frac{1}{k !} e^{\left(x-\frac{k}{2}\right) t}\left(e^{t}-1\right)^{k} \\
& =\frac{1}{k !} \sum_{j=0}^{k}\left(\begin{array}{l}
k \\
j
\end{array}\right)(-1)^{k-j} e^{\left(j+x-\frac{k}{2}\right) t} \\
& =\sum_{n=0}^{\infty}\left(\frac{1}{k !} \sum_{j=0}^{k}\left(\begin{array}{l}
k \\
j
\end{array}\right)(-1)^{k-j}\left(j+x-\frac{k}{2}\right)^{n}\right) \frac{t^{n}}{n !} .
\end{aligned}
$$

Therefore, by (2.17), we obtain the following theorem.

Theorem 2.6 For $n \geq 0$, we have

$$
\frac{1}{k !} \sum_{j=0}^{k}\left(\begin{array}{l}
k \\
j
\end{array}\right)(-1)^{k-j}\left(j+x-\frac{k}{2}\right)^{n}= \begin{cases}T(n, k \mid x), & \text { if } n \geq k \\
0, & \text { if } n<k\end{cases}
$$


Now, we observe that

$$
\begin{aligned}
e^{\left(e^{t}-1+r t\right)} & =e^{r t} e^{e^{\frac{t}{2}}\left(e^{\frac{t}{2}}-e^{-\frac{t}{2}}\right)} \\
& =\sum_{k=0}^{\infty} e^{\left(\frac{k}{2}+r\right) t} \frac{1}{k !}\left(e^{\frac{t}{2}}-e^{-\frac{t}{2}}\right)^{k} \\
& =\sum_{k=0}^{\infty} \sum_{j=0}^{\infty}\left(\frac{k}{2}+r\right)^{j} \frac{t^{j}}{j !} \sum_{m=k}^{\infty} T(m, k) \frac{t^{m}}{m !} \\
& =\sum_{k=0}^{\infty} \sum_{n=k}^{\infty} \sum_{m=k}^{n}\left(\begin{array}{c}
n \\
m
\end{array}\right) T(m, k)\left(\frac{k}{2}+r\right)^{n-m} \frac{t^{n}}{n !} \\
& =\sum_{n=0}^{\infty}\left(\sum_{k=0}^{n} \sum_{m=k}^{n}\left(\begin{array}{l}
n \\
m
\end{array}\right) T(m, k)\left(\frac{k}{2}+r\right)^{n-m}\right) \frac{t^{n}}{n !} .
\end{aligned}
$$

Therefore, by (1.10) and (2.19), we obtain the following theorem.

Theorem 2.7 For $n \geq 0$, we have

$$
\mathrm{Bel}_{n, r}=\sum_{k=0}^{n} \sum_{m=k}^{n}\left(\begin{array}{l}
n \\
m
\end{array}\right) T(m, k)\left(\frac{k}{2}+r\right)^{n-m} .
$$

From (2.18), we note that

$$
\begin{aligned}
\sum_{n=0}^{\infty} \operatorname{Bel}_{n}^{(c, r)} \frac{t^{n}}{n !} & =e^{r t} e^{\left(e^{\frac{t}{2}}-e^{-\frac{t}{2}}\right)}=\sum_{k=0}^{\infty} \frac{1}{k !} e^{\left(r-\frac{k}{2}\right) t}\left(e^{t}-1\right)^{k} \\
& =\sum_{k=0}^{\infty} \frac{1}{k !} \sum_{j=0}^{k}\left(\begin{array}{l}
k \\
j
\end{array}\right)(-1)^{k-j} e^{\left(j+r-\frac{k}{2}\right) t} \\
& =\sum_{n=0}^{\infty} \sum_{k=0}^{\infty} \frac{1}{k !} \sum_{j=0}^{k}\left(\begin{array}{l}
k \\
j
\end{array}\right)(-1)^{k-j}\left(j+r-\frac{k}{2}\right)^{n} \frac{t^{n}}{n !} \\
& =\sum_{k=0}^{\infty} \sum_{n=k}^{\infty} T(n, k \mid r) \frac{t^{n}}{n !}=\sum_{n=0}^{\infty} \sum_{k=0}^{n} T(n, k \mid r) \frac{t^{n}}{n !}
\end{aligned}
$$

Therefore, by comparing the coefficients on both sides of (2.21), we obtain the following theorem.

Theorem 2.8 For $n \geq 0$, we have

$$
\mathrm{Bel}_{n}^{(c, r)}=\sum_{k=0}^{n} T(n, k \mid r) .
$$

From (1.2), we have

$$
\sum_{n=0}^{\infty} T(n, k) \frac{t^{n}}{n !}=\frac{1}{k !}\left(e^{\frac{t}{2}}-e^{-\frac{t}{2}}+r t-r t\right)^{k}
$$




$$
\begin{aligned}
& =\sum_{l=0}^{k} \frac{(-1)^{l} r^{l}}{l !} t^{l} \frac{1}{(k-l) !}\left(e^{\frac{t}{2}}-e^{-\frac{t}{2}}+r t\right)^{k-l} \\
& =\sum_{l=0}^{k} \frac{(-1)^{l} r^{l}}{l !} t^{l} \sum_{n=k}^{\infty} T^{(r)}(n-l, k-l) \frac{t^{n-l}}{(n-l) !} \\
& =\sum_{n=k}^{\infty}\left(\sum_{l=0}^{k}\left(\begin{array}{l}
n \\
l
\end{array}\right)(-1)^{l} r^{l} T^{(r)}(n-l, k-l)\right) \frac{t^{n}}{n !} .
\end{aligned}
$$

Therefore, by comparing the coefficients on both sides of (2.23), we obtain the following theorem.

Theorem 2.9 For $n, k \geq 0$, we have

$$
T(n, k)=\sum_{l=0}^{k}\left(\begin{array}{l}
n \\
l
\end{array}\right)(-1)^{l} r^{l} T^{(r)}(n-l, k-l) .
$$

Now, we observe that

$$
\begin{aligned}
\frac{1}{k !}\left(e^{\frac{t}{2}}-e^{-\frac{t}{2}}+r t\right)^{k} & =\frac{1}{k !} \sum_{l=0}^{k}\left(\begin{array}{l}
k \\
l
\end{array}\right) r^{l} t^{l}\left(e^{\frac{t}{2}}-e^{-\frac{t}{2}}\right)^{k-l} \\
& =\sum_{l=0}^{k} \frac{r^{l}}{l !} t^{l} \sum_{n=k}^{\infty} T(n-l, k-l) \frac{t^{n-l}}{(n-l) !} \\
& =\sum_{n=k}^{\infty}\left(\sum_{l=0}^{k}\left(\begin{array}{l}
n \\
l
\end{array}\right) r^{l} T(n-l, k-l)\right) \frac{t^{n}}{n !}
\end{aligned}
$$

Therefore, by (2.1) and (2.25), we obtain the following theorem.

Theorem 2.10 For $n, k \geq 0$, we have

$$
T^{(r)}(n, k)=\sum_{l=0}^{k}\left(\begin{array}{l}
n \\
l
\end{array}\right) r^{l} T(n-l, k-l) .
$$

Let $m, k \in \mathbb{N} \cup\{0\}$. Then we have

$$
\begin{aligned}
& \frac{1}{m !}\left(e^{\frac{t}{2}}-e^{-\frac{t}{2}}+r t\right)^{m} \frac{1}{k !}\left(e^{\frac{t}{2}}-e^{-\frac{t}{2}}+r t\right)^{k} \\
& \quad=\left(\begin{array}{c}
m+k \\
m
\end{array}\right) \frac{1}{(m+k) !}\left(e^{\frac{t}{2}}-e^{-\frac{t}{2}}+r t\right)^{m+k} \\
& \quad=\left(\begin{array}{c}
m+k \\
m
\end{array}\right) \sum_{n=m+k}^{\infty} T^{(r)}(n, m+k) \frac{t^{n}}{n !}
\end{aligned}
$$


On the other hand,

$$
\begin{aligned}
& \frac{1}{m !}\left(e^{\frac{t}{2}}-e^{-\frac{t}{2}}+r t\right)^{m} \frac{1}{k !}\left(e^{\frac{t}{2}}-e^{-\frac{t}{2}}+r t\right)^{k} \\
& \quad=\sum_{l=m}^{\infty} T^{(r)}(l, m) \frac{t^{l}}{l !} \sum_{j=k}^{\infty} T^{(r)}(j, k) \frac{t^{j}}{j !} \\
& \quad=\sum_{n=m+k}^{\infty}\left(\sum_{l=m}^{n-k}\left(\begin{array}{l}
n \\
l
\end{array}\right) T^{(r)}(l, m) T^{(r)}(n-l, k)\right) \frac{t^{n}}{n !} .
\end{aligned}
$$

Therefore, by (2.27) and (2.28), we obtain the following theorem.

Theorem 2.11 For $n \geq m+k$, with $m, k \geq 0$, we have

$$
\left(\begin{array}{c}
m+k \\
m
\end{array}\right) T^{(r)}(n, m+k)=\sum_{l=m}^{n-k}\left(\begin{array}{l}
n \\
l
\end{array}\right) T^{(r)}(l, m) T^{(r)}(n-l, k) .
$$

For $m, k \geq 0$ with $m \geq k$, by (2.1), we get

$$
\begin{aligned}
\sum_{n=m}^{\infty} T^{(r)}(n, m) \frac{t^{n}}{n !} & =\frac{1}{m !}\left(e^{\frac{t}{2}}-e^{-\frac{t}{2}}+r t\right)^{m-k}\left(e^{\frac{t}{2}}-e^{-\frac{t}{2}}+r t\right)^{k} \\
& =\frac{k !(m-k) !}{m !} \frac{1}{(m-k) !}\left(e^{\frac{t}{2}}-e^{-\frac{t}{2}}+r t\right)^{m-k} \frac{1}{k !}\left(e^{\frac{t}{2}}-e^{-\frac{t}{2}}+r t\right)^{k} \\
& =\frac{1}{\left(\begin{array}{c}
m \\
k
\end{array}\right)} \sum_{l=m-k}^{\infty} T^{(r)}(l, m-k) \frac{t^{l}}{l !} \sum_{j=k}^{\infty} T^{(r)}(j, k) \frac{t^{j}}{j !} \\
& =\frac{1}{\left(\begin{array}{c}
m \\
k
\end{array}\right)} \sum_{n=m}^{\infty}\left(\sum_{l=m-k}^{n-k}\left(\begin{array}{l}
n \\
l
\end{array}\right) T^{(r)}(l, m-k) T^{(r)}(n-l, k)\right) \frac{t^{n}}{n !} .
\end{aligned}
$$

By comparing the coefficients on both sides of (2.30), we obtain the following theorem.

Theorem 2.12 For $n, m, k \geq 0$, with $n \geq m \geq k$, we have

$$
\left(\begin{array}{l}
m \\
k
\end{array}\right) T^{(r)}(n, m)=\sum_{l=m-k}^{n-k}\left(\begin{array}{l}
n \\
l
\end{array}\right) T^{(r)}(l, m-k) T^{(r)}(n-l, k) .
$$

Next, we observe that

$$
\begin{aligned}
& \frac{1}{m !}\left(e^{\frac{t}{2}}-e^{-\frac{t}{2}}+r t\right)^{m} \frac{1}{k !}\left(e^{\frac{t}{2}}-e^{-\frac{t}{2}}+r t\right)^{k} \\
& =\sum_{l=0}^{m} \frac{r^{l}}{l !} t^{l} \frac{1}{(m-l) !}\left(e^{\frac{t}{2}}-e^{-\frac{t}{2}}\right)^{m-l} \sum_{j=0}^{k} \frac{r^{j}}{j !} t^{j} \frac{1}{(k-j) !}\left(e^{\frac{t}{2}}-e^{-\frac{t}{2}}\right)^{k-j} \\
& =\sum_{n=m+k}^{\infty} \sum_{n_{1}=m}^{n-k} \sum_{l=0}^{m} \sum_{j=0}^{k}\left(\begin{array}{c}
n_{1} \\
l
\end{array}\right)\left(\begin{array}{c}
n \\
n_{1}
\end{array}\right)\left(\begin{array}{c}
n-n_{1} \\
j
\end{array}\right) r^{l+j} \\
& \quad \times T\left(n_{1}-l, m-l\right) T\left(n-n_{1}-j, k-j\right) \frac{t^{n}}{n !} .
\end{aligned}
$$


Therefore, by (2.27) and (2.32), we obtain the following theorem.

Theorem 2.13 For $n, m, k \geq 0$, with $n \geq m+k$, we have

$$
\begin{aligned}
& \left(\begin{array}{c}
m+k \\
m
\end{array}\right) T^{(r)}(n, m+k) \\
& \quad=\sum_{n_{1}=m}^{n-k} \sum_{l=0}^{m} \sum_{j=0}^{k}\left(\begin{array}{c}
n_{1} \\
l
\end{array}\right)\left(\begin{array}{c}
n \\
n_{1}
\end{array}\right)\left(\begin{array}{c}
n-n_{1} \\
j
\end{array}\right) r^{l+j} T\left(n_{1}-l, m-l\right) T\left(n-n_{1}-j, k-j\right) .
\end{aligned}
$$

Remark From (2.33) with $r=0$, we can derive the following equation:

$$
\left(\begin{array}{c}
m+k \\
m
\end{array}\right) T(n, m+k)=\sum_{l=m}^{n-k}\left(\begin{array}{l}
n \\
l
\end{array}\right) T(l, m) T(n-l, k)
$$

where $n, m, k \geq 0$ with $n \geq m+k$.

\section{Application}

A random variable $X$, taking values $0,1,2, \ldots$ is said to be a Poisson random variable with parameter $\lambda>0$ if $P(i)=P(X=i)=e^{-\lambda} \frac{\lambda^{i}}{i !}, i=0,1,2, \ldots$. Then we have $\sum_{i=0}^{\infty} P(i)=$ $e^{-\lambda} \sum_{i=0}^{\infty} \frac{\lambda^{i}}{i !}=1$. It is easy to show that the Bell polynomials $\operatorname{Bel}_{n}(x), n \geq 0$, are connected with the moments of Poisson distribution as follows:

$$
E\left[x^{n}\right]=\operatorname{Bel}_{n}(\lambda), \quad n \in \mathbb{N} \cup\{0\}, \lambda>0
$$

Let $X$ be a Poisson random variable with parameter $\lambda>0$. Then we note that

$$
\begin{aligned}
\sum_{n=0}^{\infty} E\left[(X+r \lambda)^{n}\right] \frac{t^{n}}{n !} & =e^{\lambda e^{\frac{t}{2}}\left(e^{\frac{t}{2}}-e^{-\frac{t}{2}}\right)} e^{\lambda r t} \\
& =\sum_{m=0}^{\infty} \lambda^{m} e^{\frac{m t}{2}} \frac{1}{m !} e^{\lambda r t}\left(e^{\frac{t}{2}}-e^{-\frac{t}{2}}\right)^{m} \\
& =\sum_{m=0}^{\infty} \lambda^{m} \sum_{n=m}^{\infty} \sum_{j=m}^{n}\left(\begin{array}{l}
n \\
j
\end{array}\right) T(j, m \mid \lambda r)\left(\frac{m}{2}\right)^{n-j} \frac{t^{n}}{n !} \\
& =\sum_{n=0}^{\infty}\left(\sum_{m=0}^{n} \sum_{j=m}^{n} \lambda^{m}\left(\begin{array}{l}
n \\
j
\end{array}\right) T(j, m \mid \lambda r)\left(\frac{m}{2}\right)^{n-j}\right) \frac{t^{n}}{n !} .
\end{aligned}
$$

Thus, by (3.1), we get

$$
E\left[(X+r \lambda)^{n}\right]=\sum_{m=0}^{n} \sum_{j=m}^{n} \lambda^{m}\left(\begin{array}{l}
n \\
j
\end{array}\right) T(j, m \mid \lambda r)\left(\frac{m}{2}\right)^{n-j} .
$$

From (2.6), we can derive the following equation:

$$
\begin{aligned}
& \sum_{n=0}^{\infty} \operatorname{Bel}_{n}^{(c, r)}(\lambda) \frac{t^{n}}{n !} \\
& \quad=e^{\lambda\left(e^{-\frac{t}{2}}-1\right)\left(e^{t}-1\right)} e^{\lambda\left(e^{t}-1+r t\right)}
\end{aligned}
$$




$$
\begin{aligned}
& =\left(\sum_{k=0}^{\infty}\left(\sum_{l=0}^{k} \sum_{j=0}^{l} \lambda^{l}\left(\begin{array}{l}
l \\
j
\end{array}\right)(-1)^{l-j} S_{2}\left(k, l \mid-\frac{j}{2}\right)\right) \frac{t^{k}}{k !}\right)\left(\sum_{m=0}^{\infty} E\left[(X+r \lambda)^{m}\right] \frac{t^{m}}{m !}\right) \\
& =\sum_{n=0}^{\infty}\left(\sum_{k=0}^{n} \sum_{l=0}^{k} \sum_{j=0}^{l} \lambda^{l}\left(\begin{array}{l}
l \\
j
\end{array}\right)\left(\begin{array}{l}
n \\
k
\end{array}\right)(-1)^{l-j} S_{2}\left(k, l-\frac{j}{2}\right) E\left[(X+r \lambda)^{n-k}\right]\right) \frac{t^{n}}{n !} .
\end{aligned}
$$

Thus, we have

$$
\operatorname{Bel}_{n}^{(c, r)}(\lambda)=\sum_{k=0}^{n} \sum_{l=0}^{k} \sum_{j=0}^{l} \lambda^{l}\left(\begin{array}{l}
l \\
j
\end{array}\right)\left(\begin{array}{l}
n \\
k
\end{array}\right)(-1)^{l-j} S_{2}\left(k, l \mid-\frac{j}{2}\right) E\left[(X+r \lambda)^{n-k}\right],
$$

where $X$ is the Poisson random variable with parameter $\lambda>0$, and $n \geq 0$.

\section{Conclusions}

T. Kim et al. have studied the central factorial polynomials and numbers of the second kind which are represented by some $p$-adic integrals on $\mathbb{Z}_{p}$ and investigated some properties of these numbers and polynomials. In this paper, we introduced the extended central factorial numbers and polynomials by means of generating functions, which are useful, for example, in obtaining the moments of Poisson random variables. In addition, we gave some identities for the extended central Bell polynomials in terms of those numbers and polynomials. In more detail, in Sect. 2, we investigated some properties of the extended central factorial numbers and polynomials in connection with the extended central Bell numbers and polynomials, central factorial numbers and polynomials, and central factorial numbers and polynomials of the second kind in Theorems 2.1-2.13. Furthermore, in Sect. 3, we have applied our results to the moments of Poisson distribution.

\section{Acknowledgements}

The authors would like to express their sincere gratitude to the editor and referees, who gave us valuable comments to improve this paper.

\section{Funding}

This work was supported by the National Research Foundation of Korea (NRF) grant funded by the Korea government (MEST) (No. 2017R1E1A1A03070882).

\section{Competing interests}

The authors declare that they have no competing interests.

Authors' contributions

All authors contributed equally to the manuscript and typed, read, and approved the final manuscript.

\section{Author details}

'Department of Mathematics, Kwangwoon University, Seoul, Republic of Korea. ${ }^{2}$ Department of Mathematics, Sogang University, Seoul, Republic of Korea. ${ }^{3}$ Department of Mathematics Education and ERI, Gyeongsang National University, Jinju, Republic of Korea.

\section{Publisher's Note}

Springer Nature remains neutral with regard to jurisdictional claims in published maps and institutional affiliations.

Received: 11 October 2018 Accepted: 15 January 2019 Published online: 23 January 2019

\section{References}

1. Butzer, P.L., Schmidt, M., Stark, E.L., Vogt, L.: Central factorial numbers; their main properties and some applications. Numer. Funct. Anal. Optim. 10(5-6), 419-488 (1989)

2. Carlitz, L., Riordan, J.: The divided central differences of zero. Can. J. Math. 15, 94-100 (1963)

3. Charalambides, Ch.A.: A central factorial numbers and related expansions. Fibonacci Q. 19(5), 451-456 (1981)

4. Comtet, L.: Nombres de Stirling généraux et fonctions symétriques. C. R. Acad. Sci. Paris Sér. A-B 275, 747-750 (1972) 
5. Comtet, L:: Advanced Combinatorics: The Art of Finite and Infinite Expansions. Reidel, Dordrecht (1974) (translated from the French by J.W. Nienhuys)

6. Dolgy, D.V., Kim, T.: Some explicit formulas of degenerate Stirling numbers associated with the degenerate special numbers and polynomials. Proc. Jangjeon Math. Soc. 21(2), 309-317 (2018)

7. Dolgy, D.V., Kim, T., Kwon, H.-I., Seo, J.J.: Some identities for degenerate Euler numbers and polynomials arising from degenerate Bell polynomials. Proc. Jangjeon Math. Soc. 19(3), 457-464 (2016)

8. Jang, G.-W., Kim, T., Kwon, H.-I.: On the extension of degenerate Stirling polynomials of the second kind and degenerate Bell polynomials. Adv. Stud. Contemp. Math. (Kyungshang) 28(2), 305-316 (2018)

9. Jordan, C.: Calculus of Finite Differences. Chelsea, New York (1960)

10. Kim, D.S., Kim, T.: Some identities of Bell polynomials. Sci. China Math. 58(10), 2095-2104 (2015)

11. Kim, D.S., Kim, T., Jang, G.-W.: Some identities of partially degenerate Touchard polynomials arising from differential equations. Adv. Stud. Contemp. Math. (Kyungshang) 27(2), 243-251 (2017)

12. Kim, T.: A note on ordered Bell numbers and polynomials. Adv. Stud. Contemp. Math. (Kyungshang) 27(2), 289-298 (2017)

13. Kim, T.: A note on degenerate Stirling polynomials of the second kind. Proc. Jangjeon Math. Soc. 20(3), 319-331 (2017)

14. Kim, T., Choi, J., Kim, Y.-H.: Some identities on the $q$-Bernstein polynomials, $q$-Stirling numbers and $q$-Bernoulli numbers. Adv. Stud. Contemp. Math. (Kyungshang) 20(3), 335-341 (2010)

15. Kim, T., Jang, G.-W.: A note on degenerate gamma function and degenerate Stirling number of the second kind. Adv. Stud. Contemp. Math. (Kyungshang) 28(2), 207-214 (2018)

16. Kim, T., Kim, D.S.: On $\lambda$-Bell polynomials associated with umbral calculus. Russ. J. Math. Phys. 24(1), 69-78 (2017)

17. Kim, T., Kim, D.S.: A note on central Bell numbers and polynomials. (communicated)

18. Kim, T., Kim, D.S., Jang, G.-W.: Extended Stirling polynomials of the second kind and extended Bell polynomials. Proc. Jangjeon Math. Soc. 20(3), 365-376 (2017)

19. Kwaśniewski, A.K.: On $\psi$-umbral extensions of Stirling numbers and Dobinski-like formulas. Adv. Stud. Contemp. Math. (Kyungshang) 16(1), 73-100 (2006)

20. Merca, M.: Connections between central factorial numbers and Bernoulli polynomials. Period. Math. Hung. 73(2), 259-264 (2016)

21. Roman, S.: The Umbral Calculus. Academic Press, New York (1984)

22. Zhang, W.P.: Some identities involving the Euler and central factorial numbers. Fibonacci Q. 36, 154-157 (1998)

\section{Submit your manuscript to a SpringerOpen ${ }^{\circ}$ journal and benefit from:}

- Convenient online submission

- Rigorous peer review

- Open access: articles freely available online

- High visibility within the field

- Retaining the copyright to your article

Submit your next manuscript at $\boldsymbol{~ s p r i n g e r o p e n . c o m ~}$ 